\title{
Qualificação como Médico Preceptor e a Satisfação de Seus Clientes quanto à Assistência Recebida na UBS de Origem
}

\author{
Qualifications for Medical Preceptors and User \\ Satisfaction Regarding Care Received in their \\ Basic Health Unit (BHU) of Origin
}

Cristiane Maria Carvalho Lopes ${ }^{I}$ Angélica Maria Bicudo ${ }^{2}$ Maria de Lurdes Zanolli ${ }^{I}$

\section{PALAVRAS-CHAVE}

- Educação Médica.

- Sistema Único de Saúde.

- Preceptoria.

Recebido em: 25/07/2016

Aprovado em: 01/11/2016
REVISTA BRASILEIRA DE EDUCAÇĀO MÉDICA

\begin{abstract}
RESUMO
As diretrizes curriculares propõem um perfil desejado para o médico formado, adequando o currículo às necessidades da população, com abordagem integral do indivíduo. Os profissionais da área da saúde frequentemente participam da integração ensino-serviço como preceptores. O curso de Medicina da Universidade Estadual de Campinas, em 2001, reformulou seu currículo para uma abordagem ampliada de cenários de aprendizagem, levando seus estudantes também para o ambiente em que residem seus clientes e trabalhando com a perspectiva de promover saúde. Em 2004, teve início a disciplina Atenção Integral à Saúde, em parceria com a Prefeitura Municipal de Campinas (SP), para que médicos das áreas de Pediatria, Clínica Médica e Ginecologia que atuam em Unidades Básicas de Saúde (UBS) utilizassem quatro horas de sua carga horária para supervisão de estudantes no sétimo e oitavo semestres do curso de Medicina, em conjunto com docentes da universidade. O objetivo deste estudo foi avaliar a satisfação do cliente atendido na UBS por médicos que atuam como preceptores e conhecer se estes profissionais relacionam a preceptoria com sua qualificação. De 22 preceptores, 20 aceitaram responder a uma entrevista semiestruturada sobre como se atualizam para a supervisão dos estudantes do quarto ano de Medicina da Unicamp e se, por estarem vinculados à universidade, passam por educação médica permanente, refletindo-se em um atendimento integral a seus clientes, sendo a preceptoria um instrumento para qualificar sua atuação profissional. Seus clientes foram entrevistados com um questionário fechado utilizando Smilles (emotions) para classificar o preceptor nos itens: qualidade do atendimento médico, valorização da queixa, tempo da consulta, resolutividade da consulta, confiança e aderência à orientação, e percepção geral sobre o atendimento médico. Conclusão: Mais de $90 \%$ dos clientes atendidos pelos preceptores apresentam satisfação quanto à assistência recebida e $80 \%$ dos médicos entrevistados relacionam a preceptoria como um instrumento para qualificar sua atividade profissional.
\end{abstract}




\section{KEYWORDS}

- Medical Education.

- Unified Health System.

- Preceptorship.
ABSTRACT

Medical curricula guidelines outline the desired profile for graduate physicians, adapting to the needs of the population by means of an integrated approach to the individual. Healthcare professionals often participate in initiatives integrating teaching and service as preceptors. In 2001, Unicamp Medical School reformulated its curriculum to adopt an extended approach to learning scenarios, taking their students into the environment in which their users reside and working in the aim of promoting health. In 2004, the discipline entitled "Comprehensive Healthcare" was introduced in partnership with the municipality of Campinas (SP), in order that physicians in the field of pediatrics, general practice, and gynecology working in basic healthcare units (BHU) could use four of their scheduled hours to supervise students in the seventh and eighth semesters of medical school completing preceptorships, in a joint initiative with university professors. The objective of this study was to assess the satisfaction of users served in BHUs by physicians acting as preceptors and to determine if the professionals linked the preceptorship to their qualifying as professionals. Of a total of 22 preceptors, 20 submitted to a semi-structured interview, with one of the questions asking if the preceptorship helped conceptualize the service offered to the users in the BHUs in which they were acting as physicians. Users were interviewed by means of a close-ended questionnaire using emoticons to classify the preceptor on factors including quality of medical care, appreciation of the complaint made, duration of the consultation, resolution rate, trust and adherence to orientation, and general perception of the care received. Conclusion: Over 90\% of users rated the care positively, with $80 \%$ of the physicians interviewed regarding the preceptorship as contributing to their qualifying as professionals.

\section{INTRODUÇÃO}

Com a implantação das Diretrizes Curriculares Nacionais para o curso de Medicina ${ }^{1}$, embasadas nas competências técnicas que os médicos devem adquirir para atuar com as novas tecnologias e ao mesmo tempo enfrentar os desafios do Sistema Único de Saúde (SUS), os cursos de Medicina precisaram iniciar um processo de adequação curricular com ampliação dos cenários de prática fora do hospital. As diretrizes de 2014 reforçam o modelo proposto para a formação geral do médico.

O curso de Medicina da Universidade Estadual de Campinas (Unicamp), em 2001, reformulou seu currículo para uma abordagem integral do indivíduo com a ampliação de cenários de aprendizagem e assim intensificou as atividades de seus estudantes no ambiente em que residem seus clientes com a perspectiva da formação geral para as necessidades de saúde da população.

Em 2004, o eixo longitudinal da atenção básica atinge o quarto ano da graduação com a disciplina Atenção Integral à Saúde (AIS), tendo o Programa de Incentivo às Mudanças Curriculares das Escolas Médicas (Promed) ${ }^{2}$ como parceiro no auxílio da inserção dos estudantes nas Unidades Básicas de Saúde (UBS) durante o estágio de integração ensino-serviço, promovendo a atuação na assistência aos clientes das áreas de Pediatria, Clínica Médica e Ginecologia.

Este estágio curricular é baseado na integralidade do atendimento médico, utilizando ações como: inserção do estudan- te na realidade social e sanitária da comunidade, deslocamento do hospital para Unidades Básicas de Saúde, diversificação dos cenários de aprendizagem, formação transdisciplinar e intersetorial, além de uma clínica ampliada que utiliza tecnologias leves, como a competência técnica associada a atitudes e atuação nos aspectos subjetivos e na individualidade do adoecimento ${ }^{3}$.

Para inserção dos estudantes em seis UBS (Barão Geraldo, São Quirino, Conceição, Faria Lima, Costa e Silva e Village), a universidade participa, em parceria com a Secretaria Municipal de Saúde de Campinas, da supervisão prática. Médicos que trabalham para a Prefeitura Municipal de Campinas nas especialidades de Pediatria, Ginecologia e Clínica Médica foram convidados a participar de uma seleção para atuarem em conjunto com os docentes como preceptores dos estudantes distribuídos nas diferentes UBS onde o estágio é realizado, com utilização de quatro horas de sua carga horária para supervisão de estudantes no sétimo e oitavo semestres do curso de Medicina.

A universidade contribui com o aprimoramento profissional dos preceptores com livre acesso à biblioteca, contato direto com os Departamentos e Comissão de Ensino, participação em reuniões científicas, utilização de alguns espaços de tempo para discussão de condutas médicas, promoção de encontros anuais com todos os envolvidos no ensino-aprendizado (docentes, coordenadores das disciplinas, preceptores, estudantes, gestores, coordenadores das UBS) para avaliação 
dos estágios e sugestões de melhoria e, recentemente, com a abertura da oportunidade para pós-graduação no programa Capes Ensino em 2010.

Essa responsabilidade assumida pelos preceptores quanto ao ensino-aprendizagem dos estudantes, formando médicos voltados às necessidades da população e que poderão ingressar no mercado de trabalho oferecido na atenção básica do SUS, pode ser benéfica para o profissional que já está atuando na assistência (preceptores), bem como para seus clientes ${ }^{4}$.

Os clientes do SUS modificaram seu perfil com o passar do tempo em virtude da diminuição da mortalidade infantil e melhoria da atenção perinatal, do aumento da expectativa de vida, da presença constante de doenças mais complexas decorrentes da sobrevida ${ }^{5}$ e de maior nível de exigência quanto ao atendimento prestado ${ }^{6}$. Assim, são necessários profissionais da saúde que exerçam suas atividades em UBS com competência para atender a esta demanda.

Espera-se que ser preceptor signifique posicionar este profissional em contato constante com o ensino, com a elaboração de conhecimentos que se reflitam em atendimento de qualidade aos seus clientes ${ }^{7}$.

O objetivo deste estudo foi avaliar a satisfação dos clientes quanto à assistência prestada pelos médicos preceptores em UBS de Campinas (SP) e conhecer se estes profissionais da área da saúde relacionam a preceptoria como contribuição para qualificar sua atuação.

\section{MÉTODO}

Trata-se de um estudo descritivo de delineamento transversal.

Os sujeitos da pesquisa foram médicos pediatras, clínicos gerais e ginecologistas que trabalham em Unidades Básicas de Saúde de Campinas (SP) e que supervisionam estudantes no sétimo e oitavo semestre de Medicina da Unicamp em conjunto com docentes da universidade e seus clientes, após terem concordado em participar da pesquisa e assinado o Termo de Consentimento Livre e Esclarecido (TCLE).

Este estudo foi aprovado pelo Comitê de Ética em Pesquisa da Faculdade de Ciências da Unicamp, processo no 809/2011, Conep FR-448697. Foi respeitada a Resolução 196/96 do Conselho Nacional da Saúde ${ }^{1}$.

Os preceptores foram contatados por e-mail com uma carta-convite para participarem da entrevista. Nesta solicitação, assinada pelo secretário de Saúde em exercício, receberam os aceites do Comitê de Ética da Unicamp e da Prefeitura Municipal de Campinas. Do total de 22 preceptores, 20 concordaram em participar, aceitando o convite e assinando o termo de consentimento livre. Dos preceptores entrevistados, 11 eram pediatras (dois do sexo masculino e nove do sexo feminino), quatro clínicos gerais (um do sexo masculino e três do sexo feminino) e cinco ginecologistas do sexo feminino.

Foi realizada uma entrevista com os 20 médicos por um entrevistador contratado com um roteiro de questionário semiestruturado para análise de conteúdo do perfil dos médicos/preceptores. O questionário tratava dos seguintes assuntos: como se atualizam para a supervisão dos estudantes do quarto ano de Medicina da Unicamp; e se, por estarem vinculados à universidade, passam por educação médica permanente, refletindo-se em um atendimento integral aos seus clientes, sendo a preceptoria um instrumento para qualificar sua atuação profissional. Um dos tópicos levantava a seguinte questão: “Com relação ao trabalho que o(a) Sr(a). desenvolve na UBS (Centro de Saúde), na sua opinião, ser um preceptor tem contribuído ou não para melhorar o atendimento aos seus clientes? Se sim: Tem melhorado em quais aspectos? Se não: Por que acha que não tem contribuído?"

Em seguida, fez-se contato por telefone com os preceptores participantes da pesquisa e respectivos coordenadores das UBS a fim de solicitar permissão para entrevistar seus clientes. Encaminhou-se a cada UBS uma carta de aceite do Comitê de Ética da Unicamp e da Prefeitura Municipal de Campinas.

Entre os preceptores que aceitaram participar do estudo, oito (quatro pediatras, três ginecologistas e um clínico geral) não tiveram clientes entrevistados: um não permitiu entrevistar seus clientes da UBS em que trabalhava como médico para a PMC, sete eram médicos da PMC concursados para atuarem na atenção básica, mas no momento exerciam outras funções, seja na Secretaria Municipal de Saúde de Campinas, seja trabalhando com vínculo em outra instituição (hospital universitário/ambulatório). Os 12 preceptores agendaram os horários e dias mais convenientes para a realização da entrevista nas respectivas UBS.

A entrevista aos clientes foi feita nas UBS que freqüentavam, devido à dificuldade em encontrar os domicílios. No dia estabelecido, os clientes que já estavam na UBS por um motivo particular procurando seu médico, ou seja, não foram solicitados para estar lá pela pesquisadora ou pelo preceptor, foram convidados a participar da entrevista, sendo esclarecidas suas dúvidas e respeitado o sigilo e anonimato da clientela.

Foram entrevistados 61 clientes: 40 pais ou responsáveis de sete pediatras, sendo 2 do sexo masculino e 38 femininos; Clínica Médica: 11 entrevistados de 2 preceptores, sendo 4 masculinos e 7 femininos; Ginecologia 10 entrevistados de 3 preceptores, todos do sexo feminino.

Aplicou-se aos clientes ou a seus responsáveis um questionário fechado com nove questões, todas com Smilles (emotions) para facilitar a interpretação e as respostas das perguntas. As 
questões abordavam integralidade no atendimento médico, vínculo do preceptor com a clientela, responsabilização médico-cliente e comprometimento bilateral. Cada item questionado não teve o mesmo número de alternativas, porque, como se tratava de temas diferentes, as respostas não eram condizentes com um número definido de Smilles (emotions). O total de clientes entrevistados de cada preceptor não foi o mesmo, pois a demanda e a idade da clientela são diferentes de uma Unidade Básica para outra.

\section{RESULTADOS}

O resultado, como se observa na Tabela 1, é que mais de $90 \%$ dos clientes qualificam positivamente o atendimento médico recebido.

\begin{tabular}{|c|c|c|}
\hline \multicolumn{3}{|c|}{$\begin{array}{c}\text { TABELA } 1 \\
\text { Caracterização do atendimento médico pelo } \\
\text { cliente em UBS de Campinas (SP) }\end{array}$} \\
\hline Características & $\mathbf{N}$ & $\%$ \\
\hline \multicolumn{3}{|l|}{ Qualidade do atendimento médico } \\
\hline Ótima & 44 & 72,1 \\
\hline Boa & 14 & 23,0 \\
\hline Regular & 1 & 1,6 \\
\hline Ruim & 2 & 3,3 \\
\hline \multicolumn{3}{|l|}{ Valorização da queixa do cliente } \\
\hline Sempre & 54 & 88,5 \\
\hline Algumas vezes & 5 & 8,2 \\
\hline Nunca & 2 & 3,3 \\
\hline \multicolumn{3}{|l|}{ Tempo da consulta considerado suficiente } \\
\hline Sempre & 52 & 85,3 \\
\hline Algumas vezes & 6 & 9,8 \\
\hline Nunca & 3 & 4,9 \\
\hline \multicolumn{3}{|l|}{ Resolutividade da consulta médica } \\
\hline Sempre & 52 & 85,2 \\
\hline Algumas vezes & 7 & 11,5 \\
\hline Nunca & 2 & 3,3 \\
\hline \multicolumn{3}{|l|}{ Confiança e aderência à orientação médica } \\
\hline Confiam e aderem ao tratamento & 48 & 78,7 \\
\hline Confiam, mas não aderem ao tratamento & 10 & 16,4 \\
\hline Não confiam e não aderem ao tratamento & 3 & 4,9 \\
\hline \multicolumn{3}{|l|}{ Percepção geral sobre o atendimento médico } \\
\hline Ótimo & 55 & 90,2 \\
\hline Regular & 5 & 8,2 \\
\hline Rui & 1 & 1,6 \\
\hline
\end{tabular}

Realizada a análise do resultado das entrevistas dos clientes, passou-se a estudar as entrevistas dos preceptores, sendo utilizada leitura com as recomendações de Laurence Bardin ${ }^{8}$
- exaustividade, homogeneidade, representatividade e pertinência -, com o objetivo de conhecer se os médicos relacionam a preceptoria como contribuição para a oferta de um atendimento mais qualificado aos seus clientes da UBS.

Dos 20 preceptores, 16 acreditam que sim, sendo eleitas as seguintes categorias que reforçam o resultado obtido com a clientela: troca de conhecimentos, atualização, sistematização do atendimento, comunicação e tempo de consulta.

\section{DISCUSSÃO}

O médico necessita, cada vez mais, ampliar seus conhecimentos, seja pela mudança do perfil, idade e doenças de seus clientes, seja para se adaptar ao modelo de oferta em saúde pautado na integralidade. Formar profissionais capazes para trabalhar nesse novo modelo, bem como desenvolver práticas educativas segundo este padrão não são tarefas simples ${ }^{9}$. $\mathrm{O}$ fato de ser um médico preceptor pode proporcionar mais oportunidades de atualização pelas discussões com colegas da universidade e troca de experiências.

Entrev. 3: ...o vínculo com a universidade, o conhecimento sempre crescente, as nossas reuniões de discussão de encerramento de semestre, a forma de ver a pediatria, a doença, com isso vai mudando.

Entrev. 4: ...de poder discutir algum caso de paciente meu do Centro de Saúde com os meus docentes ou com alguém que eles conheciam da Unicamp.

Entrev. 5: ...você vai ser forçado a estudar, você vai ser forçado a aprimorar seu conhecimento, a forma como você transmite o caso, a forma como você presta assistência.

Entrev. 20: Sempre contribui porque os alunos solicitam isso de você, eles têm sempre informações... e você tem que sempre estar se atualizando, porque, quanto mais você se atualiza na parte médica, mais você... se você se prepara para atender tanto ao aluno que precisa de informação quanto ao seu usuário que está ali pra ser atendido junto com ele e aí em partes você leva pra você, então onde você está...

Para se exercer a função de preceptoria em UBS, esbarra-se em obstáculos como infraestrutura precária para receber os estudantes e grande demanda nas UBS, o que limita o tempo das consultas.

Entrev. 10: Na preceptoria a gente tem a hora inteira pra ficar com o paciente, então isso é mais, isso te permite chegar a níveis mais profundos... eu vejo que tem havido um movimento pra se respeitar mais o tempo de consulta (UBS). 
Também há dificuldade na liberação para treinamento e atualizações e até falta de remuneração para tal atividade ${ }^{10,11}$. Os que se dispõem a participar de um processo de ensino-aprendizagem em conjunto com a universidade aceitam este desafio.

Em determinado momento do desenvolvimento do processo de uma pesquisa com preceptores em supervisão de apoio no estágio com estudantes, houve a curiosidade em desvelar o duplo compromisso desses profissionais médicos no desenvolvimento de suas ações de pediatra, clínico geral ou ginecologia na organização da atenção básica nas Unidades Básicas de Saúde em que desenvolvem suas atividades assistenciais.

A execução concomitante das duas tarefas, de profissional médico e preceptor, sugere que houve sinergia, um auxiliando o outro ${ }^{12}$, e sua excelente prática assistencial foi um dos fatores para escolher ser preceptor e ter sido selecionado.

Entrev. 4: ...como no ensino a gente tem que ter uma anamnese, uma preocupação com o exame físico, de fazer as coisas de uma forma completa, isso acho que se reflete no nosso dia a dia, de tentar descrever bastante o que a gente está fazendo nos prontuários dos pacientes no dia a dia, de ter essa preocupação de fazer uma avaliação completa, então eu acho que isso ajuda...

Entrev. 13: ...na minha prática, até pra poder discutir mais e melhor com os alunos... lá no Centro de Saúde onde eu fico detalhando mais, eu tenho feito uma consulta, como se diz, mais acadêmica... pra voltar àquela rotina e poder discutir com eles...

Considerando que todos devem ser preparados para a educação permanente, tanto na sua formação quanto na sua prática, cada vez é mais real a preocupação com a junção da teoria à prática no sentido da complementaridade $\mathrm{e}^{13-14}$.

A integração entre a supervisão teórico-prática do preceptor e a assistência médica no seu dia a dia nos serviços de saúde pode ser uma estratégia para a educação permanente desses profissionais ${ }^{15}$.

Entrev. 9: ...até nessa parte de atualização, ajuda bastante, você está sempre trazendo coisas novas e aplicando.

Entrev. 5: ...você tem que melhorar a linguagem que você vai usar pra passar para aquele aluno, então nesse momento você vai fazer uma série de exercícios.

O médico preceptor contribui muito para o processo de formação do estudante. Como agente do serviço, desempenha um importante papel na inserção e socialização do graduando no ambiente de trabalho, demonstrando preocupação prin- cipalmente com os aspectos de ensino-aprendizagem do desenvolvimento profissional, integrando conceitos e valores da teoria à prática ${ }^{16-17}$.

Os egressos do curso de Medicina podem chegar ao ambiente de trabalho com conhecimentos e habilidades, porém muitas vezes são exigidas deles competências, atitudes e vivências nem sempre adquiridas no processo de formação, e o preceptor tem o papel de suporte na formação para que estes tenham maior confiança e segurança quando eventualmente forem trabalhar em uma UBS. A função do preceptor está crescendo em importância, ao mesmo tempo em que o ambiente de trabalho e a satisfação da clientela estão em constante mudança, com a exigência de que o novo profissional esteja sempre atualizado.

Entrev. 16: ...porque ele exige mesmo que você fique o tempo todo estudando, revendo temas que às vezes a gente acaba deixando passar em algum momento...

Segundo Donabedian ${ }^{18}$, os resultados esperados pelo atendimento dependem da oferta de ações em saúde, que repercutem tanto no indivíduo como na população. Verifica-se que a satisfação do cliente envolve uma multiplicidade de fatores, como acesso, estrutura física e organizacional, bem como a relação com o médico ${ }^{19}$. Sua avaliação é subjetiva, refletindo sua visão do conceito de qualidade e expectativa desejada. Como observado nas entrevistas, os clientes se satisfazem com o tempo de consulta ofertado pelo médico, e este, ao exercer a preceptoria, percebe que há necessidade de um tempo maior, a fim de poder ser mais resolutivo. As condições de saúde desejada e a satisfação estão muito relacionadas ao nível socioeconômico, mas mesmo assim nos oferecem dispositivos para gerar ações no campo de prevenção e promoção à saúde que devem ser priorizadas ${ }^{6}$. Esta discussão deve ser realizada na prática com os estudantes que devem ser formados para as necessidades da população.

O preceptor deve ter a capacidade de integrar os conceitos e valores da instituição de ensino superior e do trabalho-serviço, ajudando o profissional em formação a desenvolver estratégias possíveis para resolver os problemas cotidianos da atenção à saúde $\mathrm{e}^{20-21}$.

A seleção realizada pela Faculdade de Ciências Médicas da Unicamp para preceptores atuarem na disciplina de Atenção Integral à Saúde com estudantes no sétimo e oitavo semestre do curso de Medicina teve êxito no que se refere à qualidade da assistência que prestam nos serviços. São profissionais avaliados pela maioria dos clientes como fazendo atendimentos médicos de ótima qualidade. Estes dados corroboram os estudos realizados por Gouveia et al. ${ }^{6}$ e Souza et al. ${ }^{22}$. 
A frequência alta de ótimas avaliações dos médicos pelos clientes pode sugerir que os estudantes estão tendo uma assessoria de profissionais competentes e compromissados com os clientes e que acreditam que a dupla atuação enriquece sua atuação.

Entrev. 6: ...do contato com a universidade, de ir à própria pessoa que, porque como você se dispõe a ficar com os alunos, você acaba procurando... relembrar as coisas que você já tinha deixado de fazer na sua prática, reler coisas, procurar saber mais sobre as doenças, fazer diagnóstico, até pra discussão com os alunos...

Acredita-se que se deva investir na capacitação para o desenvolvimento da preceptoria nesses profissionais, o que é de extrema importância para a formação de graduandos na área da saúde. Por estarem em contato com a universidade, esses profissionais têm mais oportunidades de atualização, propiciando uma oferta de atenção à saúde mais efetiva e com maiores níveis de satisfação da clientela.

\section{REFERÊNCIAS}

1. Brasil. Ministério da Educação. Conselho Nacional de Educação. Resolução CNE/CES nº 4, de 07/11/2001. Diretrizes Curriculares Nacionais do Curso de Graduação em Medicina. Brasília: Câmara de Educação Superior; 2001.

2. Brasil. Ministério da Saúde. PROMED- Programa de Incentivo às Mudanças Curriculares para as Escolas Médicas. Brasília: MS; 2002.

3. Nogueira MI. As Mudanças na Educação Médica Brasileira em Perspectiva: Reflexões sobre a Emergência de um Novo Estilo de Pensamento. Rev Bras Educ Med. 2009; 33(2):262-70.

4. Barreto VH, Monteiro RO, Magalhães GS, Almeida RC, Souza LN. Papel do Preceptor da Atenção Primaria em Saúde na Formação da Graduação e Pós-Graduação da Universidade Federal de Pernambuco um Termo de Referência. Rev Bras Educ Med. 2011; 35(4):578-83.

5. Lavras C. Atenção primária à saúde e a organização de redes regionais de atenção à saúde no Brasil. Saúde e Sociedade 2011; 20(4):867-74.

6. Gouveia GC, Souza WVD, Luna CF, Souza-Júnior PRBD, Szwarcwald CL. Satisfação dos usuários do sistema de saúde brasileiro: fatores associados e diferenças regionais. Rev Bras Epidemiol 2009; 12(3):281-96.

7. Lavras C. Atenção primária à saúde e a organização de redes regionais de atenção à saúde no Brasil. Saúde e Sociedade 2011; 20(4):867-74.

8. Bardin L. Análise de Conteúdo. Lisboa, Portugal; 70 Edições, LDA, 2009.
9. Oliveira Rodrigues AAA, Juliano IA, Melo MLC, Prestes FC. Processo de Interação Ensino, Serviço e Comunidade: a Experiência de um PET. Saúde. Rev Bras Educ Med 2012; 361(1 supl 2):184-92.

10. Trajman A, Assunção N, Venturi M, Tobias D, Toschi W, Brant V. A preceptoria na rede básica da Secretaria Municipal de Saúde do Rio de Janeiro: opinião dos profissionais de Saúde. Rev Bras Educ Med.; 33(1):24-32.

11. Da Silva VC, Viana LDO, Santos CRGC. The preceptorship in undergraduate nursing: na integrative review of the literature. Revista de Pesquisa: Cuidado é Fundamental Online 2013; 5(5):20-28.

12. Hsu HY, Lee LL, Fu CY, Tang CC. Evoluation of a leadership orientation program in Tawan: Preceptorship and leader competencies of the newnurse mananger. Nurse Education Today 2011; 31(8):809-14.

13. Bispo EPF, Tavares CHF, Tomaz JMT. Interdisciplinaridade no ensino em saúde: olhar do preceptor na saúde da Família. Interface (Botucatu) 2014; 18(49):337-350.

14. Rodrigues AMM, Freitas CHA, guerreiro MGS, Jorge MSB. Preceptoria na perspectiva da integralidade: conversando com enfermeiros. Rev Gaúcha Enferm 2014; 35(2):337-350.

15. Rodrigues CDS. Competências para a Preceptoria: Construção no Programa de Educação pelo Trabalho para a Saúde. Porto Alegre; 2012. Mestrado [Dissertação] — Universidade Federal do Rio Grande do Sul.

16. Mills JE, Francis KL, Bonner A. Mentoring, clinical supervision and preceptoring: clarifying the conceptual definitions for Australian rural nurses. A review of the literature. Rural Remote Health. 2005; 5(3):410.

17. Botti SHO, Rego S. Preceptor, supervisor, tutor e mentor: quais são seus papéis? Rev Bras Educ Med. 2008; 32(3):363-73.

18. Donabedian A. The definition of quality: a conceptual exploration. Explorations are quality and approaches to its assessment. Ann Arbor, Michigan: Heath Administration Press 1988; 3-31.

19. Esperidião M, Trad LAB. Avaliação de satisfação de usuários. Cienc Saude Colet 2005; 10(supl.0):303-12.

20. Quill TE. A cross-sectional study of the influence of the ambulatory preceptor as a role model. Arch Intern Med 1987; 147: 971-973.

21. Armitage P, Burnard P. Mentors or preceptors? Narrowing the theory-practice gap. Nurse Educ Today 1991; 11(3):225229.

22. Souza LM, Maranhão LC, Oliveira KM, Figueiredo LS, De Morais Rodrigues D, Pires CAA. Perfil dos usuários atendidos em uma Unidade Básica de Saúde Ananindeua (Pará-Brasil). Ciência \& Saúde. 2011; 4(2):50-58. 


\section{CONTRIBUIÇÃO DOS AUTORES}

Cristiane Maria Carvalho Lopes realizou este trabalho como parte da dissertação de mestrado no Programa de Pós-Graduação em Clínica Médica - Área de Concentração Ensino e Saúde, UNICAMP, sob a orientação da Prof. (a) Dr. (a) Angélica Maria Bicudo e Coorientação da Prof. (a) Dr. (a) Maria de Lurdes Zanolli, que também participaram na análise dos dados, redação e revisão do artigo.

\section{CONFLITO DE INTERESSES}

Os autores declaram não haver conflito de interesses.

\section{ENDEREÇO PARA CORRESPONDÊNCIA}

Cristiane Maria Carvalho Lopes

Universidade Estadual de Campinas

Faculdade de Ciências Médicas

Rua Alexander Fleming, 126

Cidade Universitária Zeferino Vaz - Campinas

CEP 13083-970 - SP

E-mail: cris_pedi@hotmail.com; angelicabicudo@gmail.com; zanolli@fcm.unicamp.br 